\title{
Epithelial-Myoepithelial Carcinoma of External Auditory Canal Evolving from Pleomorphic Adenoma
}

\author{
Jae-Wook Lee ${ }^{1}$, Na-Hye Myung ${ }^{2}$ and Myung-Whan Suh ${ }^{1}$ \\ ${ }^{1}$ Departments of Otolaryngology-Head \& Neck Surgery, ${ }^{2}$ Pathology, Dankook University College of Medicine, Cheonan, Korea
}

$\begin{array}{ll}\text { Received } & \text { July 13, 2012 } \\ \text { Revised } & \text { September 29, } 2012 \\ \text { Accepted } & \text { October 16, 2012 }\end{array}$

Address for correspondence

Myung-Whan Suh, MD

Department of Otolaryngology-

Head \& Neck Surgery,

Dankook University

College of Medicine,

201 Manghyang-ro, Dongnam-gu,

Cheonan 330-715, Korea

Tel +82-41-550-3974

Fax $+82-41-556-1090$

E-mail drmung@naver.com
A 45-year-old female patient visited the clinic due to ear fullness. A $8 \mathrm{~mm}$ sized mass was found in the external auditory canal. It was surgically removed and the pathology exam confirmed epithelial-myoepithelial carcinoma (EMC). EMC is glandular epithelial neoplasm of the salivary gland. EMC originating from the external auditory canal is very rare, and only 1 case has been reported so far in the literature. The difference between our case and the one previously published was that the tumor was previously proven as a benign tumor (i.e. pleomorphic adenoma) 5 years ago. Therefore, this is the first case in the literature showing the evolvement of pleomorphic adenoma of the external auditory canal into EMC.

Korean J Audiol 2012;16:148-151

KEY WORDS: External auditory canal cancer · Epithelial-myoepithelial carcinoma.

\section{Introduction}

Epithelial-myoepithelial carcinoma (EMC) is a rare glandular epithelial neoplasm of the salivary gland, with an incidence of less than 1 percent of all salivary gland tumors. EMC generally occurs in the parotid gland, but some cases in the maxillary sinus, nasal cavity, trachea, larynx and lacrimal gland have been reported. EMC originating from the external auditory canal is very rare and only one case has been reported so far worldwide. ${ }^{1)}$ We have recently experienced a case of EMC of the external auditory canal in a 45-year-old female patient. The difference between our case and the one previously published was that the tumor was previously proven as a benign mass 5 years ago. However, it seems that pleomorphic adenoma of the external auditory canal can also evolve into EMC, similar to the location in the salivary gland. This is the first case in the literature showing the evolvement of pleomorphic adenoma of the external auditory canal to EMC.

\section{Case Report}

A 45-year-old women visited the hospital for right ear fullness, which started a month before the visit. She had a histo- ry of tumor resection for pleomorphic adenoma in the right external auditory canal 5 years ago. She did not have any particular past medical history, except the above-mentioned surgery, nor any particular findings in family history or social history. On the visit, her general condition was relatively fine, and an $8 \mathrm{~mm}$ large pink soft tissue lump was observed on physical examination above the right external auditory canal (Fig. 1). On temporal bone CT, an $8 \times 6 \mathrm{~mm}$ large mass of soft tissue density lesion confined to the external auditory canal was observed as well. Under the diagnosis of recurrent pleomorphic adenoma, resection was performed with $2 \mathrm{~mm}$ margin around the mass. However, the results of postoperative permanent biopsy were consistent with EMC (Fig. 2). The tumor showed relatively low-grade differentiation, and there was no invasion of the resection margin. The estimated prognosis based on salivary gland tumor was expected to be very favorable. Few weeks after the operation, granulation tissue appeared at the surgical site, and external auditory canal MRI showed a $5 \times 6 \mathrm{~mm}$ nonenhancing soft tissue lesion in the right external auditory canal. However, invasion into surrounding tissue or erosion of the external auditory canal bone was not detected. Positron emission tomography computed tomography showed a hypermetabolic local lesion in the external auditory canal due to 
inflammation, but no invasion into the surrounding tissue or distant metastasis was observed (Fig. 3). The pathological slides from the first operation 5 years ago were reviewed again to rule out the possibility of misdiagnosis, but there was no evidence of malignancy at that time.

Although the mass had been completely removed, we were concerned about potential microscopic seeding of the EMC. A modified form of lateral temporal bone resection with tympanic membrane preservation was performed. The posterosuperior bone canal and adjacent soft tissue were completely removed, but the anteroinferior bone canal and tympanic membrane were preserved. The classical lateral temporal bone resection involves removal of the bony ear canal, tympanic membrane, and the middle ear, which leads to a high degree of conductive hearing loss. However, we expected good prognosis in this case, such as low grade salivary glands tumor. Therefore a modified form of lateral temporal bone resection was chosen in order to preserve the hearing.
According to the pathologic findings, there was no remaining malignant tumor, and only chronic inflammation was observed. Two months after the operation, the temporal bone CT showed no abnormal contrast enhancement suggestive for a new mass or the recurrence of carcinoma in the external auditory canal. The surgical site was maintained satisfactorily without any sign of recurrence, and the patient's hearing was well preserved for 6 months after the operation (Fig. 4).

\section{Discussion}

EMC is a rare tumor first described in 1972 by Donath, et al., ${ }^{2)}$ and around 320 cases have been so far reported worldwide. The primary site was the parotid gland in $62 \%$ of the cases, and EMC is also distributed in the nasal cavity, sinuses, palate, submandibular gland, lesser salivary gland, larynx and trachea. ${ }^{3-5)}$ The average age of tumor onset is 61 years and the incidence is higher in women. ${ }^{3,6)}$ EMC originating from the
Fig. 1. Preoperative temporal bone CT, endoscopic image and pure tone audiogram. A $8 \times 6 \mathrm{~mm}$ sized mass lesion was identified in the right superior external auditory canal. A minimal air-bone gap was identified on the pure tone audiogram.
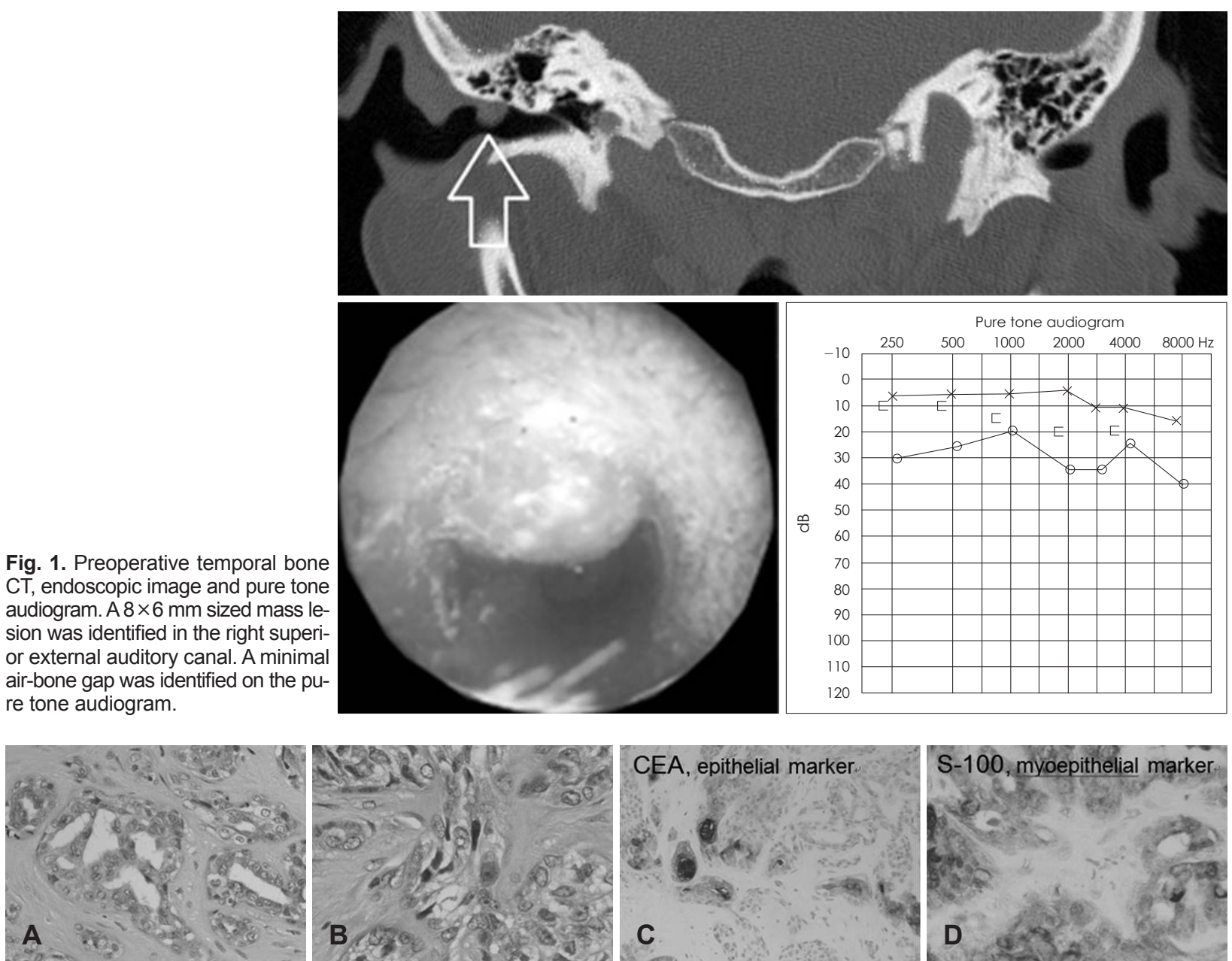

Fig. 2. Histologic findings of the epithelial myoepithelial carcinoma. The tumors are pathologically characterized by two types of cells, showing low-cuboidal and dark epithelial cells in the inner rows or parts of the nests $(A)$ and polygonal, plump and clear myoepithleial cells in the outer parts (B). Carcinoembryonic antigen (CEA) is an epithelial marker (C) and S-100 protein is a myoepithelial marker that highlights the cytoplasms of the tumor cells with myoepithelial differentiation (D). 
external auditory canal has been reported so far in only one case worldwide. ${ }^{1)}$ Our report is presumably the second case in the literature.

The diagnosis of EMC is based on optical microscopy, and definite diagnosis is made through immunohistochemical stu-
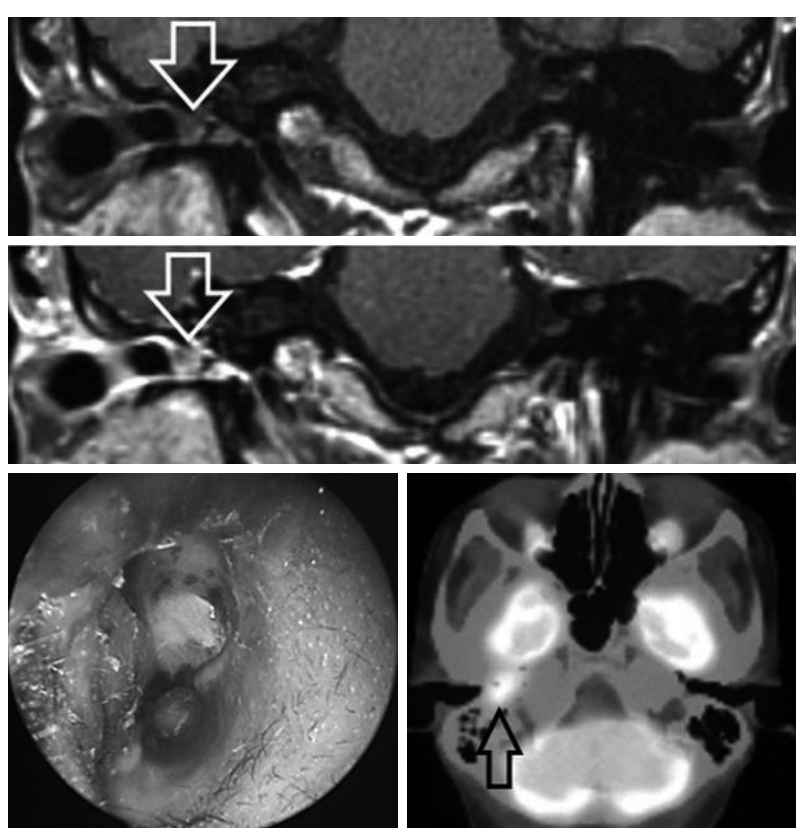

Fig. 3. A month after mass excision, IAC MRI T1, T1 enhance, endoscopic image and PET-CT. A $5 \times 6 \mathrm{~mm}$ sized non-enhancing soft tissue lesion was identified in the right superior external auditory canal. Little evidence of significantly focal hypermetabolic lesion around right external auditory canal. IAC MRI: internal auditory canal magnetic resonance imaging, PET-CT: positron emission tomography computed tomography. dy. ${ }^{7)}$ Histologically, EMC is characterized by tubules consisting of two types of cells, i.e. eosinophilic cuboidal epithelial cells in the inner layer and myoepithelial cells surrounding the membrane with plasma in the outer layer. In immunohistochemical test, epithelial cells in the inner layer can be detected through cytokeratin staining, and myoepithelial cells in the outer layer through antibody staining with S-100 protein and $\alpha$ smooth muscle actin. ${ }^{8)}$ As presented in Fig. 2, we were also able to observe the typical cytokeratin staining inner layer and S100 protein staining outer layer. EMC needs to be differentiated from clear cell predominant tumors, such as clear cell myoepithelioma, acinic cell carcinoma and adenoid cystic carcinoma. However, it is easily confused, particularly because adenoid cystic carcinoma has a double-layer structure. S-100 protein is usually staining the outer layer of EMC, but the inner layer of the adenoid cystic carcinoma.

One interesting finding is that the EMC of this patient was previously proven to be a benign tumor (i.e. pleomorphic adenoma) 5 years ago. As pleomorphic adenoma can evolve into carcinoma ex pleomorphic adenoma in the salivary gland, ${ }^{9)}$ we presumed that the EMC of this patient has evolved from the former pleomorphic adenoma. Malignant transformation of pleomorphic adenoma is logically possible, but it has never been identified in the external auditory canal. Therefore, this is the first report in the literature presenting the evolvement of pleomorphic adenoma into EMC in the external auditory canal.

Another issue is identifying from which cell the EMC has originated. In the report of Kong, et al., ${ }^{1)}$ the authors have pre-
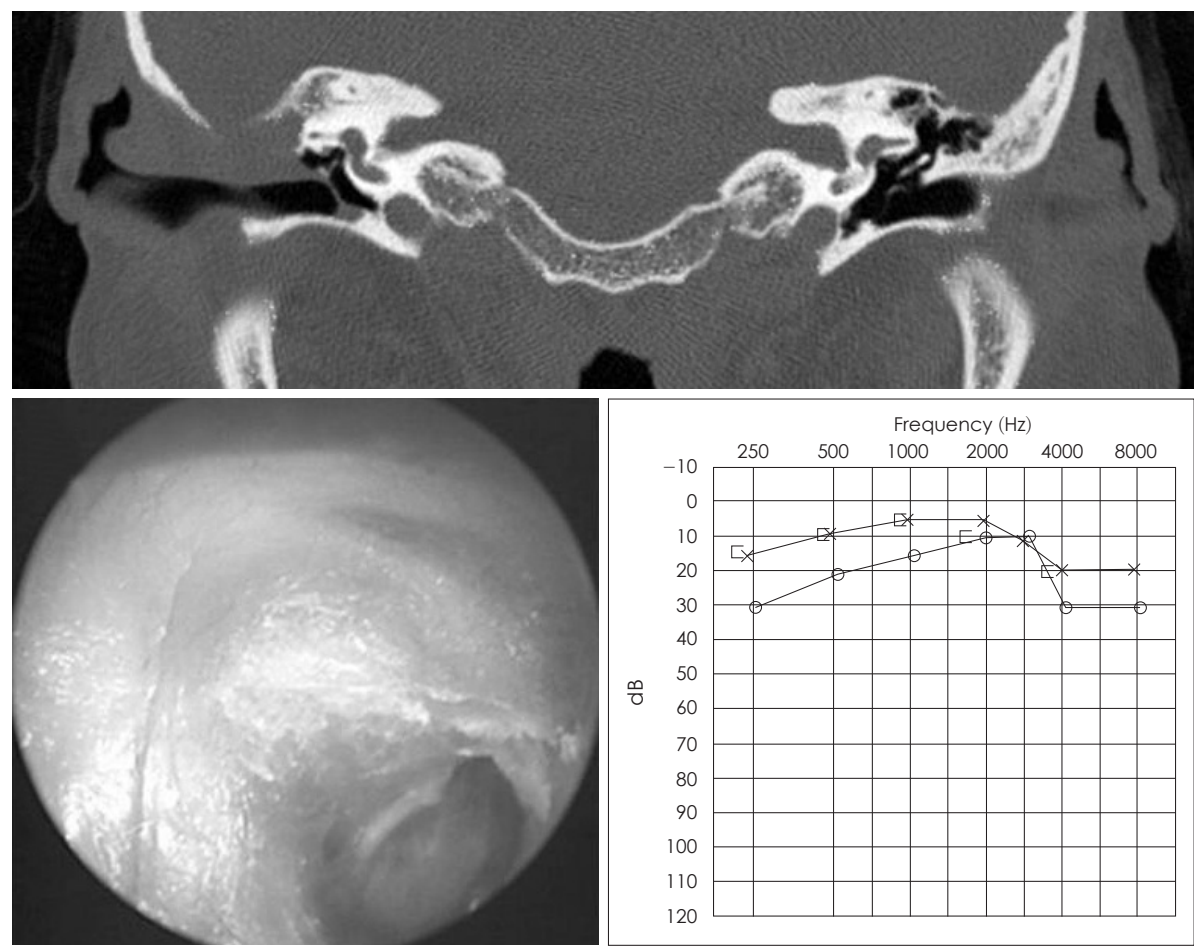

Fig. 4. After modified lateral temporal bone resection operation temporal bone $\mathrm{CT}$, endoscopic image and pure tone audiogram. Wound healed well and there was no evidence of tumor recurrence at 6-month follow up. Also, hearing was preserved. 
sumed that the cancer has originated from an ectopic minor salivary gland located in the external auditory canal skin. This hypothesis is feasible, but another possibility may be that the EMC has originated from the eccrine cells of the external auditory canal. Ectopic minor salivary gland may arise anywhere in the head and neck region, but the chances of minor salivary gland arising from the external auditory canal may be low. Considering that the normal eccrine gland is also composed of epithelial and myoepithelial cells, eccrine gland can also be an origin of EMC. Although not certain, we thought that it was more likely that the EMC arised from the eccrine gland than the ectopic minor salivary gland of the external auditory canal.

Pleomorphic adenoma is a mixed tumor that is composed of epithelial and mesenchymal components. Pleomorphic adenoma frequently arises from the salivary gland, but it may also rarely arise from ectopic salivary gland tissue or from sebaceous glands of the skin. If it arises from the sebaceous glands, it is known as "chondroid syringoma or mixed tumor". However, histologically Pleomorphic adenoma arising from the sebaceous gland and from the salivary gland are the same. ${ }^{10)}$ While pleomorphic adenoma in the skin is a rare tumor, it can be located in various sites in the head and neck, such as the scalp, eyelids, nose, cheek and upper lip. In the external ear, the external auditory canal is a common site of occurrence. ${ }^{11)}$ There are those who support the view that their histologic origins are in the ceruminal glands of the cartilaginous segment of the EAC. $^{12)}$

Because EMC is a rare disease, the standard treatment method has not been established yet. Nevertheless, surgical resection is the best method known. Vascular or lymphatic invasion, tumor necrosis, anaplasia of myoepithelium and clear debridement are crucial factors for prognosis. ${ }^{13)}$ Local recurrence is relatively common, reported in around $31.3 \%$ of cases. ${ }^{3)}$ EMC is classified as a low-grade tumor and it requires long-term follow-up. ${ }^{14)}$ In our case, pathologic findings showed no sign of poor prognosis, such as vascular or lymphatic invasion, tumor necrosis, anaplasia of myoepithelium and tumor invasion of the resection margin. Based on the experience in salivary gland tumors, the prognosis was expected to be favorable. Thus, a typical lateral temporal bone resection was not performed. In order to conserve the hearing, we performed a modified form of lateral temporal bone resection, preserving the tympanic membrane. This surgical method is generally identical with the typical lateral temporal bone resection, except that it maintains the eardrum and the anteroinferior part of the bone ca- nal. In this patient, the mass had already been completely removed in the first operation and histological findings were very favorable. Consequently, after in-depth consultation on the pro and cons of the operation, a limited form of operation was performed.

Thus, we report a patient with histologically rare EMC of the external auditory canal. It is presumed that pleomorphic adenoma of the external auditory canal has evolved into EMC, as in salivary gland tumors.

\section{REFERENCES}

1) Kong SK, Goh EK, Chon KM, Lee IW. Epithelial-myoepithelial carcinoma in the external auditory canal. Otolaryngol Head Neck Surg 2008:139:598-9.

2) Donath K, Seifert G, Schmitz R. [Diagnosis and ultrastructure of the tubular carcinoma of salivary gland ducts. Epithelial-myoepithelial carcinoma of the intercalated ducts]. Virchows Arch A Pathol Pathol Anat 1972;356:16-31.

3) Batsakis JG, el-Naggar AK, Luna MA. Epithelial-myoepithelial carcinoma of salivary glands. Ann Otol Rhinol Laryngol 1992;101:540-2.

4) Horinouchi $H$, Ishihara T, Kawamura M, Kato R, Kikuchi K, Kobayashi K, et al. Epithelial myoepithelial tumour of the tracheal gland. J Clin Pathol 1993;46:185-7.

5) Mikaelian DO, Contrucci RB, Batsakis JG. Epithelial-myoepithelial carcinoma of the subglottic region: a case presentation and review of the literature. Otolaryngol Head Neck Surg 1986;95:104-6.

6) Corio RL, Sciubba JJ, Brannon RB, Batsakis JG. Epithelial-myoepithelial carcinoma of intercalated duct origin. A clinicopathologic and ultrastructural assessment of sixteen cases. Oral Surg Oral Med Oral Pathol 1982;53:280-7.

7) Cheung FM, Hioe F, Kong JH. Histologic variant of the epithelialmyoepithelial carcinoma of the salivary gland: a case report. Head Neck 1995;17:437-44.

8) Michal M, Skálová A, Simpson RH, Rychterová V, Leivo I. Clear cell malignant myoepithelioma of the salivary glands. Histopathology 1996;28:309-15.

9) Suh MW, Hah JH, Kwon SK, Jung YH, Kwon TK, Kim KH, et al. Clinical manifestations of recurrent parotid pleomorphic adenoma. Clin Exp Otorhinolaryngol 2009;2:193-7.

10) Nishimura S, Murofushi T, Sugasawa M. Pleomorphic adenoma of the auricle. Eur Arch Otorhinolaryngol 1999;256:22-4.

11) Nardone M, Ferrara G, Nappi O, Di Maria D, Villari G. [Pleomorphic adenoma in unusual anatomic sites: case reports and review of literature]. Acta Otorhinolaryngol Ital 2002;22:158-63.

12) Markou K, Karasmanis I, Vlachtsis K, Petridis D, Nikolaou A, Vital V. Primary pleomorphic adenoma of the external ear canal. Report of a case and literature review. Am J Otolaryngol 2008;29:142-6.

13) Seethala RR, Barnes EL, Hunt JL. Epithelial-myoepithelial carcinoma: a review of the clinicopathologic spectrum and immunophenotypic characteristics in 61 tumors of the salivary glands and upper aerodigestive tract. Am J Surg Pathol 2007;31:44-57.

14) Kasper HU, Mellin W, Kriegsmann J, Cheremet E, Lippert H, Roessner A. Epithelial-myoepithelial carcinoma of the salivary gland--a low grade malignant neoplasm? Report of two cases and review of the literature. Pathol Res Pract 1999;195:189-92. 